\title{
Pulse generators based on air-insulated linear-transformer-driver stages
}

\author{
B. M. Kovalchuk, A. V. Kharlov, E. V. Kumpyak, and A. A. Zherlitsyn \\ Institute of High-Current Electronics, 2/3 Academichesky Avenue, 634055, Tomsk, Russia
}

(Received 27 September 2012; published 16 May 2013)

\begin{abstract}
In this paper we present the design and test results of pulse generators based on air-insulated lineartransformer-driver stages that drive a vacuum transmission line. A custom designed unit, referred to as a capacitor block, was developed for use as a main structural element of the transformer stages. It incorporates two capacitors GA $35426(40 \mathrm{nF}, 100 \mathrm{kV})$ and a multichannel multigap gas switch. Two types of stages were developed: (1) stage LTD-20 with four modules in parallel and five capacitor blocks in each module (in tests of this stage current amplitude up to $850 \mathrm{kA}$ with $\sim 140 \mathrm{~ns}$ rise time was obtained on a $0.05 \Omega$ load at $100 \mathrm{kV}$ charging voltage); (2) stage LTD-4 with two modules in parallel and two capacitor blocks in each module. Several installations were built on the base of these stages, including a linear transformer, consisting of two identical LTD-20 stages in series, and a high power electron accelerator on the base of LTD-4 stages. The design, tests results, and main problems are presented and discussed in this paper for these installations.
\end{abstract}

DOI: 10.1103/PhysRevSTAB.16.050401

PACS numbers: $84.30 . \mathrm{Ng}$

\section{INTRODUCTION}

Linear-transformer-driver (LTD) technology was pioneered by the Institute of High Current Electronics in Russia more than a decade ago [1]. LTD based drivers are currently considered for many applications, including high-current $Z$-pinch drivers [2], medium current drivers for isentropic compression experiments [3], and relatively low current accelerators for radiography and $X$ pinches [4]. The LTD driver is an induction generator similar to a linear induction accelerator (LIA), an inductive voltage adder (IVA), and a linear pulsed transformer (LPT). Detailed review on the induction generators is given by Smith [5]. All induction generators are based on Faraday's law, which states that the time-varying magnetic flux $B(t)$ penetrating a loop generates at the ends of this loop a voltage difference $U(t)$ equal to $U(t)=S d B / d t$, where $S$ is the cross section of the loop. In the induction generator normally the loop is realized as a pillbox-shaped toroid, which is usually called "an induction cavity." This toroid has a continuous azimuthal gap, and the voltage $U(t)$ appears across this gap. Since historically the first devices utilizing Faraday's law were called "transformers," all induction generators can also be considered as transformers. The LIA, IVA, and LPT can be formed by one or a series of identical induction cavities. In the LIA a central bore is threaded by a beam of propagating particles which gets accelerated as they drift through each of the cavity gaps. The IVA is also formed by a series of identical induction cavities, but unlike the LIA, its bore is threaded by a center conductor that together with

Published by the American Physical Society under the terms of the Creative Commons Attribution 3.0 License. Further distribution of this work must maintain attribution to the author(s) and the published article's title, journal citation, and DOI. the cylindrical surface of the bore forms an output line that connects to the load.

Similar to the IVA and LPT, the LTD driver consists of several induction cavities called "LTD stages" plus the center electrode threading through the axis of the cavities. The main difference from the LIA, IVA, and LPT induction cavities is that the LTD stage encloses its primary capacitive energy storage. Simplified schematics of an LTD assembly is presented in Fig. 1. It may be seen that the capacitors $(C)$, switches $(S)$, and ferromagnetic cores $(F)$ are directly incorporated into the LTD stages to generate a fast output voltage pulse which is added along a vacuum coaxial line. Intermediate pulse forming elements are not required in the LTD technique, which means the LTD driver is more compact compared to other generators having similar output parameters. One more advantage is that the body of the LTD cavity is held under ground potential during the shot and it allows one to assemble the cavities in series or in parallel depending on load requirements. Pulse length and current amplitude of the LTD assembly are defined mainly by parameters of one stage, and the output voltage is directly proportional to the number of stages (NV0).

Typically a primary side of the induction cavities is filled with transformer oil for additional electrical isolation of the components [6,7]. It is often not desirable to use oil and it especially can cause problems in large assemblies. In this paper we present new design of LTD stages, which can be used as modular elements in design of multi-MV and multiMA generators, with voltage or current addition in assembly of such stages with connecting in series or in parallel. Dry air is used both as insulation in the stages and as working gas in the LTD spark gap switches.

Two types of air-insulated stages were developed: (1) stage LTD-20 with four modules in parallel and five 


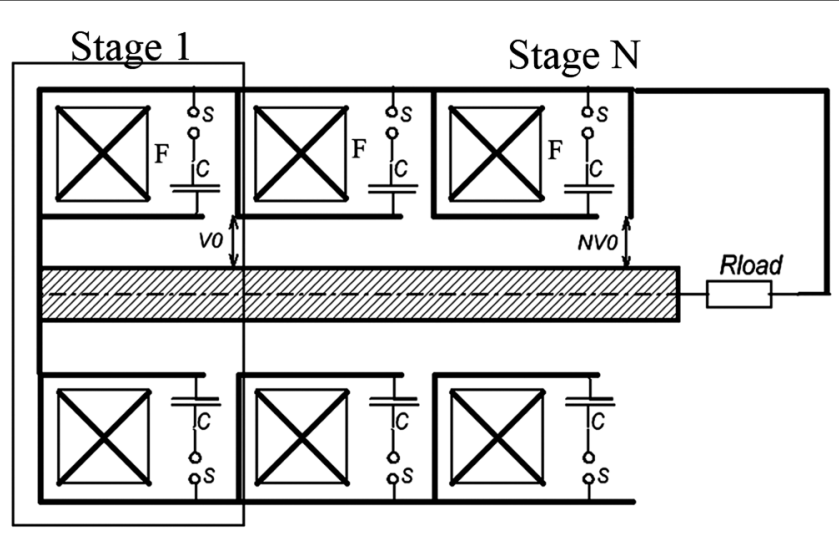

FIG. 1. Principal schematics of the LTD assembly.

capacitor blocks in each module [8]; (2) stage LTD-4 with two modules in parallel and two capacitor blocks in each module [9]. There are a few main improvements in the new stages in comparison with the earlier developed ones: (1) Dry air at atmospheric pressure is used both as insulation in the generator and as a working gas in the spark gap switches. (2) Charging voltage and stored energy density are increased due to implementation of new capacitors and improvement of the switch technology. (3) Modular configuration, employed in the LTD design, ensures easy handling and maintenance (each module can be taken out without disassembling of the whole installation).

\section{EXPERIMENTAL SETUP}

\section{A. Capacitor block}

A capacitor block is the main structural element of the transformer stages. The design of the capacitor block is given in Figs. 2(a)-2(c). It incorporates two capacitors GA $35426(40 \mathrm{nF}, 100 \mathrm{kV})$ and a multichannel multigap gas switch. Body (1) of the block is formed from epoxy compound. Capacitors (2) are sealed within epoxy in the block body permanently. Epoxy sealing enhances external electric insulation of the capacitors and provides the possibility of operation at $100 \mathrm{kV}$ charging voltage in atmospheric air. Inner capacitor terminals are connected with a switch high voltage (HV) electrode (4). The multichannel gas switch was developed for use in the capacitor block. It is a plane geometry switch with seven gaps in one channel and six channels in parallel. The switch [Fig. 2(b)] is assembled on an epoxy slab (3). The switch contains 36 ball electrodes (ball bearings steel, $22.3 \mathrm{~mm}$ in diameter). Voltage divider (9), assembled from film resistors on $68 \mathrm{M} \Omega$, provides voltage distribution between rows of electrodes. A triggering pulse is supplied to the second row of the ball electrodes through capacitive coupling between the triggering cable (7) and the ball electrodes. At arrival of the triggering pulse with polarity, opposite to the main pulse, homogeneous voltage distribution is distorted and the switch gaps breaks down sequentially. The capacitor block has been
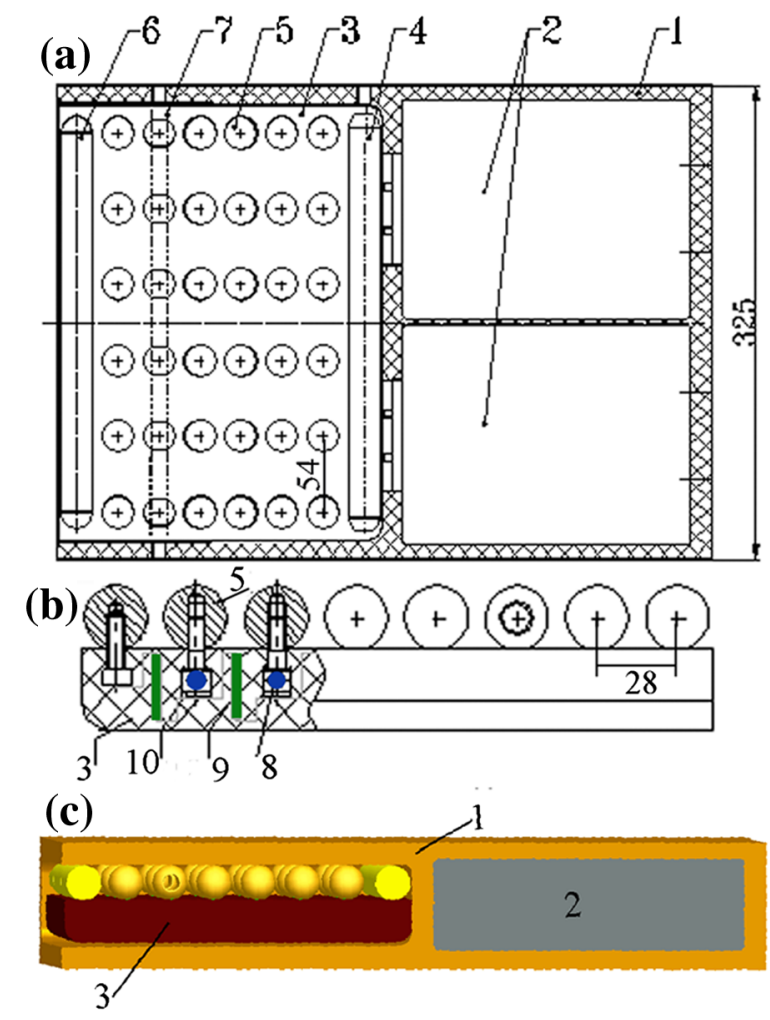

FIG. 2. (a) Cross-section drawing of the capacitor block, (b) electrode system assembly, (c) 3D model cut view. Here shown: 1-epoxy body; 2-capacitors GA 35426; 3-switch plate; 4-HV electrode; 5-ball switch electrodes; 6-low voltage electrode; 7-triggering cable; 8-conductive cord; 9-divider resistors; 10 - special screws for the balls mounting.

previously investigated and tested [10,11]. A twodimensional simulation of real switch geometry with a back current grounded electrode has been performed for $100 \mathrm{kV}$ charging voltage. Figure 3 demonstrates that the electric field increases to the last electrode up to $29 \mathrm{kV} / \mathrm{cm}$ due to impact of the grounded back current electrode. Selfbreakdown voltage for the gap of $5.7 \mathrm{~mm}$ between the

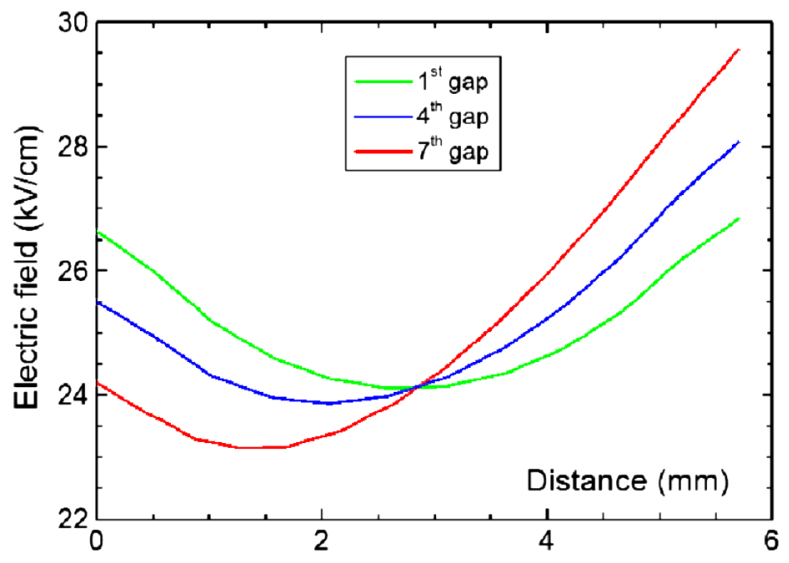

FIG. 3. Field distribution in the interelectrode gaps for the standard switch configuration at equal distribution of potentials on the electrodes. 
spherical electrodes with the diameter of $22.3 \mathrm{~mm}$ is $\sim 18-19 \mathrm{kV}$ in atmospheric air (experiment), which corresponds to $31.5-33.3 \mathrm{kV} / \mathrm{cm}$ of an average electric field strength in the gap.

\section{B. Stage LTD-20}

The LTD-20 transformer stage is assembled from four modules, connected in parallel on the LTD primary. The stage design is shown in Fig. 4. The modules (1) are assembled on four sides of a basement plate (2) and fixed to it from the low voltage side. An acrylic insulator is placed between the modules and plate (2). A ferromagnetic stage inductor is mounted on the combined electrodes (4) around a support ring (3). Combined electrodes (4) and the stage basement are fixed to the support ring (3). The stage inductor (position 5, Fig. 3) consists of three cores, which are wound up by transformer steel (ET-3425, saturation field $B s=1.9 \mathrm{~T}$, reset field $B r=1.4 \mathrm{~T}$, electrical resistivity $0.5 \times 10^{6} \Omega \times \mathrm{m}$ ) strip of $18 \mathrm{~mm}$ width and $50 \mu \mathrm{m}$ thickness, with insulating $10 \mu \mathrm{m}$ mylar tape between layers. Length of the center core line is $\sim 4.78 \mathrm{~m}$, the total number of turns is $\sim 1215$. The cross-section area of the stage inductor cores is $\sim 33 \mathrm{~cm}^{2}$; the saturation value of a voltage-time product for the stage inductor is $\sim 11 \mathrm{mV} \mathrm{s}$. The front and rear surfaces of the cores are covered by epoxy compound.

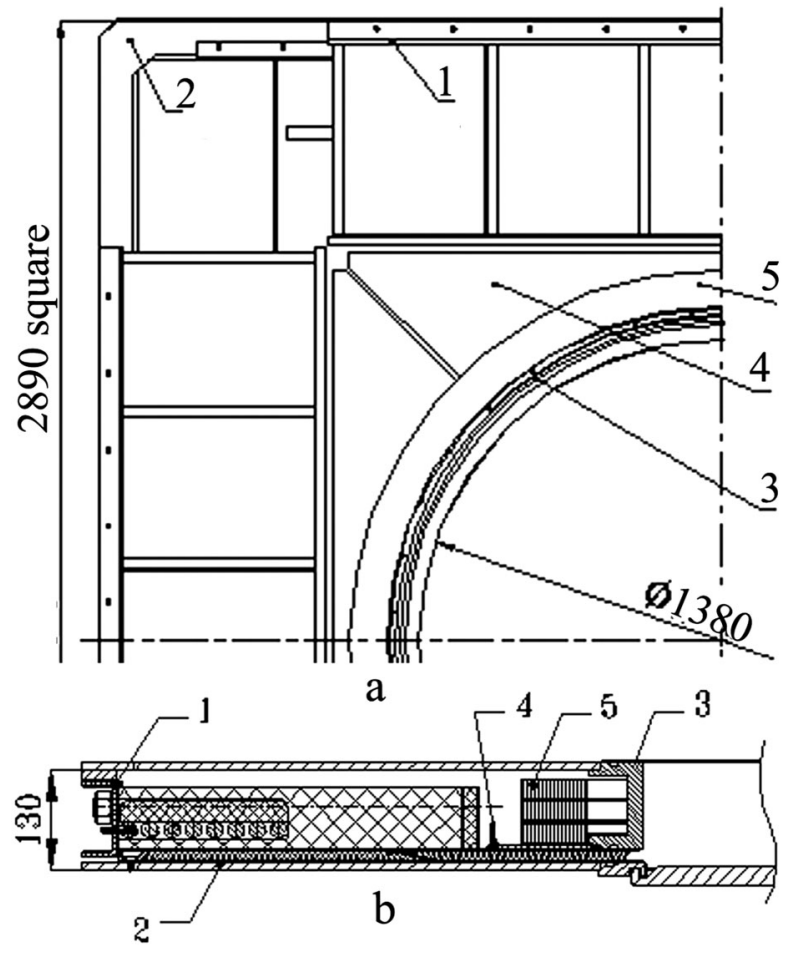

FIG. 4. LTD-20 stage design: (a) top view of one quarter of the stage; (b) partial cross section through central axis. Here are: 1-module of the LTD stage; 2-basement plate; 3-support ring; 4-combined electrodes; 5-stage inductor core (all dimensions are given in $\mathrm{mm}$ ).
A module of the transformer stage is assembled from five capacitor blocks, connected in parallel. Blocks of the module are fixed between a basement from a steel channel and dielectric plate. The triggering pulse is applied from both sides of the module in order to reduce time delay in triggering between blocks. Stage dimensions are shown in Fig. 3. The weight of the LTD-20 stage is $\sim 1200 \mathrm{~kg}$. In tests of this stage current amplitude up to $850 \mathrm{kA}$ with $\sim 140 \mathrm{~ns}$ rise time was obtained on the $0.05 \Omega$ load at $100 \mathrm{kV}$ charging voltage.

In the linear transformer drivers (as shown in Fig. 1), the cavity walls represent the single-turn winding around the core. When the pulsed voltage is applied to the single-turn winding that surrounds the core (actually the cavity walls), it generates the eddy current in the magnetic core, which together with inductance of the core determine the total current flowing along the cavity walls and the energy loss due to generation of the eddy current. The stage was tested in two regimes: (1) maximum output parameters of the stage were derived at the opened circuit of the stage inductor (it corresponds to removal of parasitic inductor circuit A2 on Fig. 6), when all the current flows through the load; (2) at the connection of the outer conductor of the stage coaxial (diameter of $1380 \mathrm{~mm}$ ) with the outer perimeter of the stage basement, the stage inductor circuit is connected in parallel with the load. This regime corresponds to operation of the transformer stage, incorporated in a LTD assembly.

The impact of the stage inductor on the output pulse parameters was investigated with the $0.1 \Omega$ load. The losses on the cores magnetization, current leakage in the stage inductor circuit, and volt-second integral were estimated in these experiments. In the first case, without leakage through the stage inductor circuit, amplitude of the load current is $\sim 619 \mathrm{kA}$, current rise time up to maximum is $\sim 130 \mathrm{~ns}$, and amplitude of the load voltage is $\sim 62 \mathrm{kV}$. The energy, delivered to the load, mounts to $\sim 7.3 \mathrm{~kJ}$ from $8 \mathrm{~kJ}$ stored in the capacitors. Maximum load power is $\sim 38 \mathrm{GW}$. At the closed circuit of the stage inductor the load power and energy decreased to $\sim 36.5 \mathrm{GW}$ and $6.6 \mathrm{~kJ}$, respectively. The difference in load energy for two variants provides experimental estimation of energy losses in the stage inductor $\sim 700 \mathrm{~J}$. Test results with $0.1 \Omega$ load are summarized in Table I.

TABLE I. Pulse parameters depending on charging voltage. Load $0.1 \Omega-0.8 \mathrm{nH}$, circuit of the stage inductor is opened/ closed. $U 0$ - charging voltage of the stage; $I$-amplitude of a load current; $T f$-current rise time; $U$-amplitude of the load voltage; $P$-load power; $W$-load energy; $\eta$-efficiency of transfer of the stored energy to the load.

\begin{tabular}{lcccccc}
\hline \hline$U 0, \mathrm{kV}$ & $I, \mathrm{kA}$ & $T f, \mathrm{~ns}$ & $U, \mathrm{kV}$ & $P, \mathrm{GW}$ & $W, \mathrm{~kJ}$ & $\eta, \%$ \\
\hline 100 & $619 / 607$ & 130 & $62 / 60.5$ & $38.1 / 36.6$ & $7.3 / 6.6$ & $91 / 82$ \\
95 & $586 / 573$ & 130 & $58.5 / 57$ & $34.1 / 32.6$ & $6.5 / 5.9$ & $90 / 82$ \\
90 & $549 / 538$ & 130 & $55 / 53.5$ & $30.0 / 28.8$ & $5.8 / 5.3$ & $90 / 82$ \\
\hline \hline
\end{tabular}




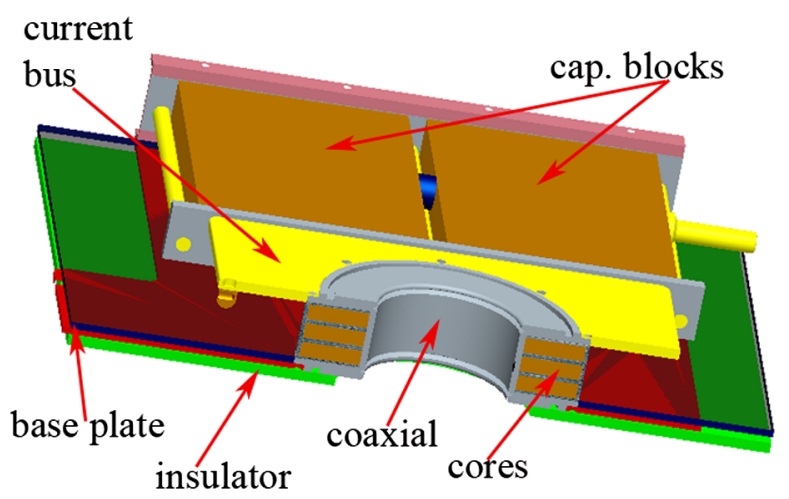

FIG. 5. Half cut view of LTD-4 stage with main elements shown.

\section{Stage LTD-4}

A 3D cut view of the LTD-4 transformer stage is given in Fig. 5. The stage consists of two modules, connected in parallel on a primary turn of the linear transformer, and stage inductor, consisting of three cores.

The module of the transformer stage is assembled from two capacitor blocks, connected in parallel. The modules are placed on a base plate symmetrically relatively to the plate center and fixed to the plate from low voltage side. High voltage terminals of the capacitor blocks are connected with the combined current bus through spring contacts. Acrylic insulator sheets (15 mm thickness) are used as insulators between stages. Acrylic insulator sheets $(10 \mathrm{~mm}$ thickness) are also used for insulation between the modules and base plate. Stage overall dimensions are $750 \times 1510 \times$ $132 \mathrm{~mm}^{2}$. The inductor of the transformer stage is installed outside of the outer conductor of a vacuum coaxial line. The stage inductor consists of three cores, assembled in the polyethylene body. The cores are wound in the same fashion as for the LTD-20 stage. The length of the stage inductor middle core line is $\sim 1 \mathrm{~m}$, the cross-section area of the stage inductor cores is $\sim 40 \mathrm{~cm}^{2}$; the saturation value of a voltage-time product for the stage inductor is $\sim 13 \mathrm{mV} \mathrm{s}$.

Two electron accelerators have been built on the base of this stage: (a) a compact accelerator from six LTD-4 stages for small-scale experiments [9] and (b) a high-current electron-beam accelerator for pumping of a $\mathrm{Xe}_{2}$ lamp with 24 stages LTD-4 [11].

\section{ELECTRICAL MODEL}

Simulations of the two LTD pulse generators driving inductive-resistive loads (resistor Rload and inductance Lload connected in series) have been performed using PSpice, a circuit-modeling code. The simulation model is described in detail in [10]. Figure 6(a) shows an equivalent electrical scheme of a discharge circuit for the two LTD-20 stages assembly (any number of stages can be simulated). A1 represents 20 capacitor blocks, connected in parallel, each of which is modeled as indicated by Fig. 6(b). Here $C c=80 \mathrm{nF}$ and $R c \approx 0.05 \Omega$ are block capacitance
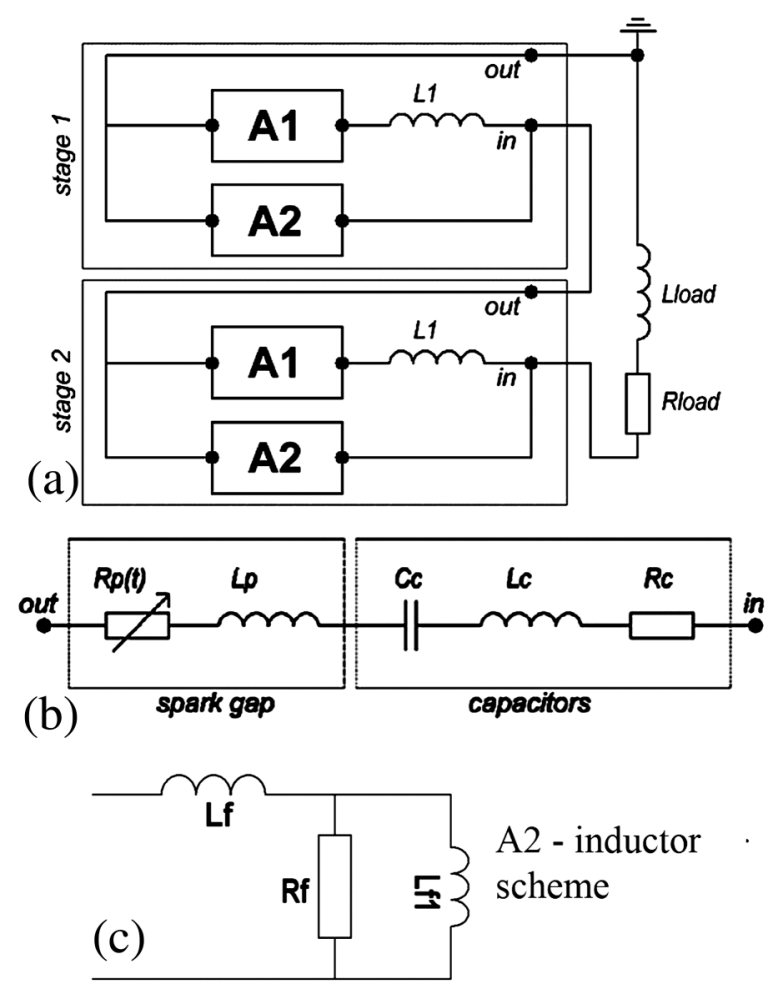

FIG. 6. Simulation network: (a) electrical scheme of the capacitor block $\left(C_{C}, L_{C}\right.$, and $R_{C}$ are capacitor parameters; $R_{P}(t)$ and $L_{P}$ are electrical parameters of the switch); (b) equivalent electrical scheme for the two LTD-20 stages assembly; (c) equivalent scheme for the stage inductor modeling.

and active resistance, respectively; $L c \approx 30 \mathrm{nH}$ stands for equivalent inductance of one capacitor block in the stage. This value was derived from experiments with one capacitor block. $R_{P}(t)$ and $L_{P}$ are the dynamic resistance and inductance of the multichannel switch. L1 is equivalent inductance of a transmission line between the stage and load; Lload and Rload are the load inductance and active resistance.

A2, modeled as indicated by Fig. 6(c), represents the inductance and resistance of the primary turn. This inductance can be partitioned on the part of the stage inductor contour with air gap $\mathrm{Lf}$ and that due to the magnetic cores Lf1.

Resistance $R f$ in the scheme of Fig. 6(c) provides energy loss in cores, corresponding to the experimental value. Inductance $\mathrm{Lf}$ is estimated from the stage inductor geometry. Inductance Lf1 is also estimated from the stage inductor geometry, but taking into account relative magnetic permeability of the cores. Our simulation model implies that the relative magnetic permeability of the cores $\mu$ change stepwise from constant value $\mu 1$ to $\mu 2$ at the moment when the volt-second integral reaches saturation value. Mean relative magnetic permeability of the cores in a nonsaturated state can be evaluated as $\mu 1 \sim 2000 \tau$, where $\tau$ is a pulse rise time in $\mu$ s. Mean relative magnetic 
permeability of the multilayered cores in a saturated state is $\mu 2 \sim 4-7$.

Values of the inductances and resistances in the scheme of Fig. 6 and more accurate values for $\mu 1, \mu 2$ have been derived from our preliminary experiments on the stage prototype. Voltage and current in the stage inductor circuit have been measured in those experiments. Satisfactory agreement between calculated and experimental curves for voltage and current has been obtained at $R f \sim$ 0.9 Ohm, $\mu 1 \sim 300, \mu 2 \sim 4$.

Switch resistance $R s(t)$ and inductance $L p(t)$ in our model are simulated with use of the Braginskii model [12], which implies hydrodynamic expansion of a spark channel in approximation of constant conductivity. According to this model, transition resistance of a spark gap is defined as

$$
R s(t)=\frac{d[\mathrm{~cm}]}{k\left(\int I^{2 / 3} d t\right)},
$$

where $d$ is total interelectrode gap in a switch, $k$ is constant, which depends on the sort of working gas, pressure, and electrical conductivity of the channel. For air at atmospheric pressure specific electrical conductivity is $\sigma \sim 300(\Omega \mathrm{cm})^{-1}$, constant $k \approx 1.2 \times 10^{5}$ [13].

Inductance $L p$ was estimated from geometric dimensions of a switch channel. Channel radius $r(t)$ temporal dependence is calculated according to the model

$$
r(t)=r(t 0)+\left(\frac{k}{\pi \sigma}\right)^{\frac{1}{2}}\left(\int_{t 0}^{t}|i(t)|^{0.67} d t\right)^{\frac{1}{2}},
$$

where initial radius channel (at the beginning of a spark phase) is taken as $r(t 0) \sim 0.1 \mathrm{~mm}$.

\section{INSTALLATIONS}

\section{A. Linear transformer on base of LTD-20 stages}

The transformer consists of two LTD-20 stages, connected in series [14]. The total capacitance is equal to $1.6 \mu \mathrm{F}$. Linear transformer stores $16 \mathrm{~kJ}$ of energy at $100 \mathrm{kV}$ charging voltage. The block scheme for the test bed of two connected in series LTD stages is given in Fig. 6(a). Scheme operation proceeds as follows: Capacitor blocks of the first and second stages are charged from a power supply up to given voltage. At firing of a premagnetization generator on command from a remote control device the premagnetization current flows through inductors of the first and second stages. Synchropulse from the premagnetization generator triggers a delay pulse generator, which with time delay $\sim 60 \mu$ s fires a main triggering generator. The main triggering generator is fired at the moment of maximum current in the stages inductors. Pulses from the triggering generator come to the switches of the capacitor blocks and trigger these switches. At the switches firing capacitors of the blocks are discharged on primary turns of the stages and the current flows in a load
Rload, included in the secondary turns of the stages. A digital oscilloscope registers voltage on the stages inductors, load voltage, triggering voltage, and load current. All registered signals are acquired and saved in a computer for analysis and storage.

A 3D view of the assembled linear transformer without the top cover is shown in Fig. 7(a) [picture in Fig. 7(b)]. The transformer footprint is $290 \times 295 \mathrm{~cm}^{2}$; the height is $278 \mathrm{~mm}$ (without auxiliary equipment). A requirement for installation of the decoupling resistors between capacitor blocks in a module appears from the possibility of a switch self-fire in one of the modules. At self-fire of one of the blocks at $100 \mathrm{kV}$ in one of the modules, if the module is installed in the transformer from two connected in series stages (load, for example, $0.1 \mathrm{Ohm}$ and $2.6 \mathrm{nH}$ ), current in the switch can be increased up to $130 \mathrm{kA}$, energy loss to $\sim 500 \mathrm{~J}$. Our experience during tests revealed that at such currents in the switch channel and energy losses in the switch a damage of the capacitor block body is possible. In order to decrease energy, released in the capacitor block switch at its self-fire, decoupling resistors $(\mathrm{NiCr}$ wire, $\sim 100 \Omega$ ) were installed between capacitor blocks in all modules.

Tests procedures.-At first, eight modules have been tested separately on a special test bed at operation on the
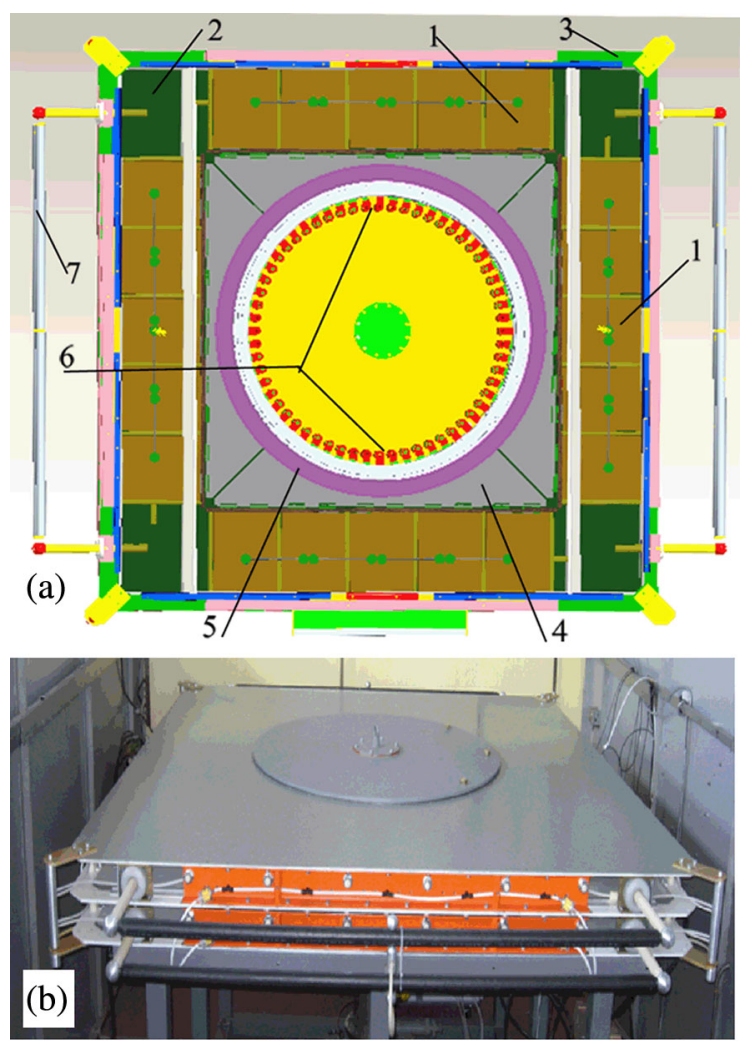

FIG. 7. 3D view of the assembled linear transformer without top cover (a), picture of the installation (b): 1-modules; 2insulation; 3-stage plate; 4-combined electrode; 5-magnetic cores; 6-load resistors; 7—charging resistors. 
TABLE II. Results of investigations of the linear transformer from two stages. Here $I_{L}$ is current amplitude, $T_{f}$ is rise time, $U_{L}$ is load voltage, $P_{L}$ is load power, $W_{L}$ is load energy, and $\eta$ is efficiency of the energy transfer to a load.

\begin{tabular}{|c|c|c|c|c|c|c|}
\hline \multirow[b]{2}{*}{ Load } & \multicolumn{2}{|c|}{$\begin{array}{l}2.51 \mathrm{nH} \\
0.073 \Omega\end{array}$} & \multicolumn{2}{|c|}{$\begin{array}{l}2.66 \mathrm{nH} \\
0.098 \Omega\end{array}$} & \multicolumn{2}{|c|}{$\begin{array}{c}3.06 \mathrm{nH} \\
0.14 \Omega\end{array}$} \\
\hline & Experiment & Simulation & Experiment & Simulation & Experiment & Simulation \\
\hline$I_{L}, \mathrm{kA}$ & 902 & 942 & 836 & 837 & 694 & 702 \\
\hline$T_{f}, \mathrm{~ns}$ & 168 & 148 & 166 & 144 & 158 & 138 \\
\hline$U_{L}, \mathrm{kV}$ & 65,6 & 71 & 80,3 & 83 & 103 & 99 \\
\hline$U_{L}$ at $d I / d t=0$ & 64 & 69 & 78,8 & 82 & 102 & 98 \\
\hline$P_{L} \mathrm{GW}$ & 58 & 66 & 66 & 69 & 71 & 69 \\
\hline$W_{L}, \mathrm{~kJ}$ & 11 & 10 & 12 & 11 & 13 & 12 \\
\hline$\eta, \%$ & 68,7 & 62,5 & 75 & 69 & 81 & 75 \\
\hline
\end{tabular}

load $0.2 \mathrm{Ohm}$ and $\sim 6.4 \mathrm{nH}$ inductance. Charging voltage at those tests started from $80 \mathrm{kV}$ and rose up to $100 \mathrm{kV}$. Stable operation of the modules without breakdowns is obtained after $\sim 50-100$ shots (the switches electrodes are conditioned). Further operation proceeds without any breakdowns at charging voltage $100 \mathrm{kV}$. The total amount of shots on all modules was about 1400 in those tests.

Then tests of two connected in series stages have been carried out with three types of the load at $95 \mathrm{kV}$ charging voltage. One shot was made in 6 min. After 630 shots the installation has been disassembled and all modules were examined. At revision of all modules it has been found that there were cracks in bodies of three capacitor blocks. New capacitor blocks were manufactured. In order to decrease energy, released in the capacitor block switch at its self-fire ( $\sim 15$ self-fires were observed in 630 shots), decoupling resistors were installed between the capacitor blocks in the modules. 500 shots were made after mounting of the decoupling resistors without failures in the capacitor blocks. The total amount of shots at tests of the modules was 3234; at tests of the linear transformer there were 1100 .

The transformer has been tested on resistive-inductive loads. It was demonstrated in the experiments that $\sim 80 \%$ of the energy, stored in the stages capacitors, is delivered to a load with maximum power in the pulse $\sim 72 \mathrm{GW}$. Simulation of the linear transformer operation was performed with the model, described above. Results of experiments and calculations are given in Table II. Reasonable agreement between experiment and simulation was obtained (discrepancy between simulation results and experiment is $\sim 5 \%$ in the current amplitude and $\sim 10 \%$ in the current rise time). The standard deviation in current and voltage amplitudes did not exceed 5\%.

\section{B. Electron accelerator on base of LTD-4 stages}

A high-current electron-beam accelerator was developed [15] for pumping of a $\mathrm{Xe}_{2}$ lamp for the hybrid femtosecond $\mathrm{XeF}(\mathrm{C}-\mathrm{A})$ laser system. It is intended for injection of the electron beam into the cylindrical gas cavity (diameter of $400 \mathrm{~mm}$, length of $1600 \mathrm{~mm}$, the absolute pressure up to 3 bars). A picture and an assembling drawing of the electron accelerator are given in Figs. 8 and 9. Two HV pulse generators, made on a scheme of a linear transformer with vacuum electric insulation of a secondary turn, are used to drive an electron diode. Each generator consists of 12 LTD-4 stages. The HV pulse generators are placed on both sides of the vacuum chamber 4 , where the vacuum electron diode and pumped gas volume are installed. Transformer stages are connected in series and hermetically sealed in between through the acrylic insulators (position 3, Fig. 9). A drawing of this insulator is given in Fig. 10. It is made from acrylic sheet with dimensions $1436 \times 990 \times 15 \mathrm{~mm}$ and with a machined central hole as shown in Fig. 10. The central conductor (6), placed inside the stages, serves as part of a secondary turn for all 12 LTD stages. One end of this conductor is connected with the

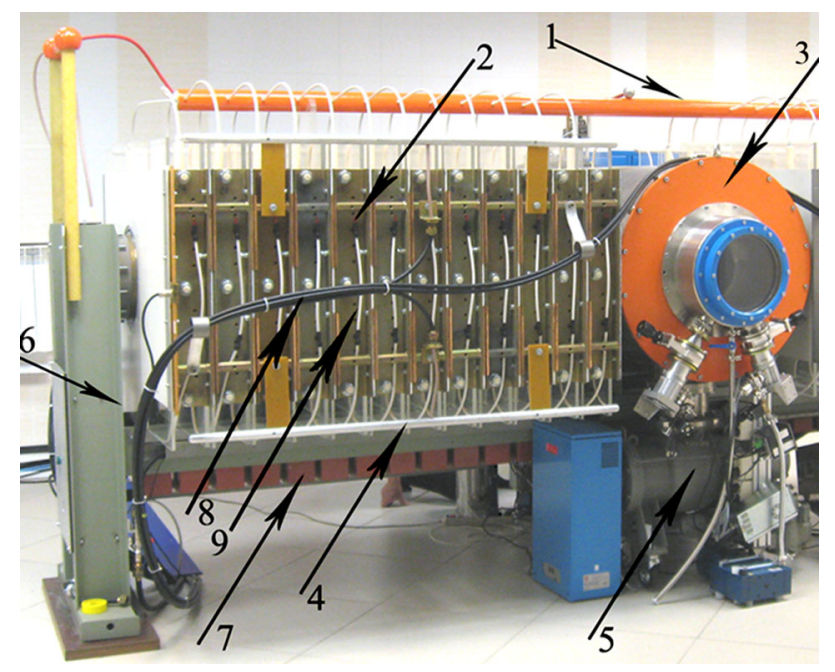

FIG. 8. Partial view of the generator (one LTD and central vacuum chamber): 1-charging bus; 2-transformer stage; 3-vacuum chamber; 4-triggering bus; 5-vacuum pump; 6-support; 7-capacitors of the premagnetization block; 8-triggering cables; 9-air purge system of the transformer stage switches. 


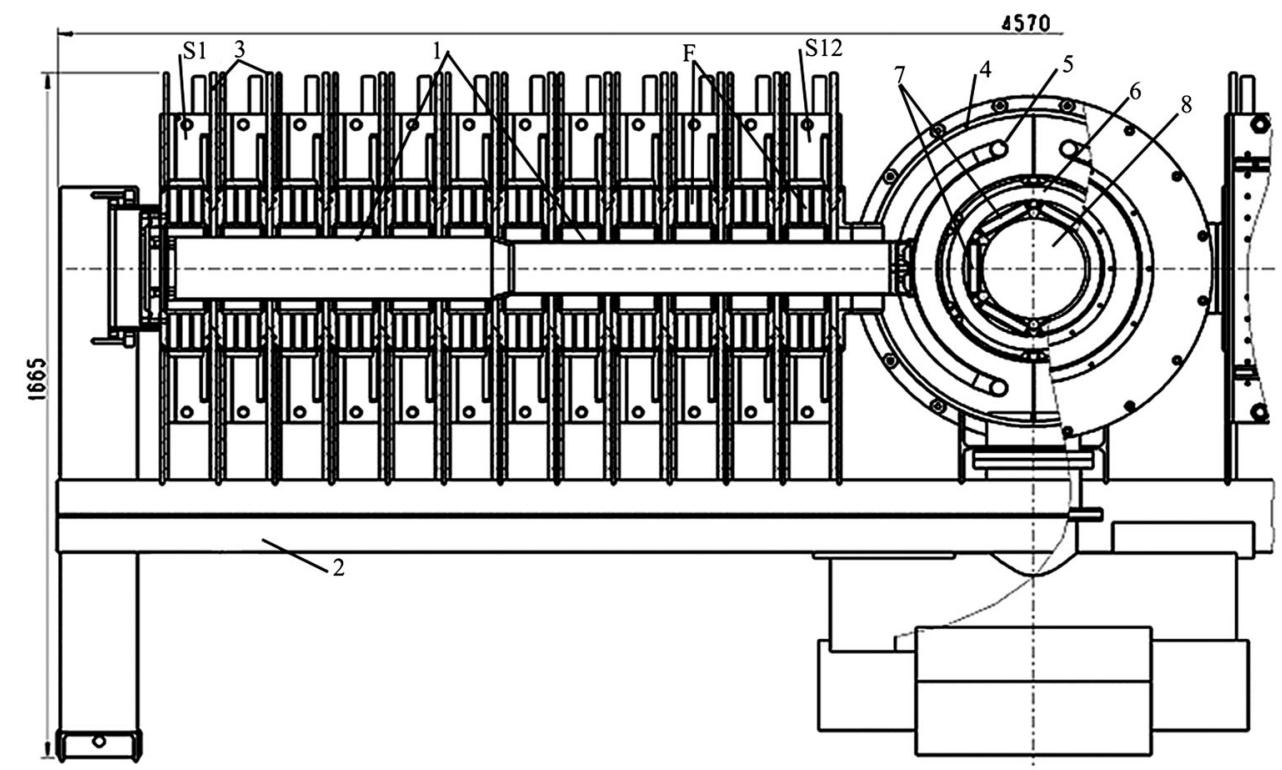

FIG. 9. Assembly drawing of the accelerator: 1-central conductor; 2-basement; S1-S12-12 LTD-4 stages; F-ferromagnetic cores; 4-body of the vacuum chamber; 5 -cathode; 6 -Xe volume; $7-\mathrm{CaF}_{2}$ windows of a laser cell; 8-laser cell.

cathode of the electron diode, the other one is fixed on a flange of the first stage of the linear transformer. A central conductor (6) is made as a cylinder with variable diameter of $160 / 140 \mathrm{~mm}$. High voltage is localized here in the vacuum coaxial, and vacuum insulation of a secondary turn directly means that electric field strength on the central conductor 6 is below vacuum breakdown level and magnetic insulation is not necessary. Except measurement of the diode current, the output voltage on each stage $U 1-U 12$, current in the inductor of the stage Ii and current in the beginning of coaxial line $I 0$ were registered in the experiments. Registered signals were used for calculation of diode voltage $U d$, diode resistance $\mathrm{Rd}$, beam power $P$,

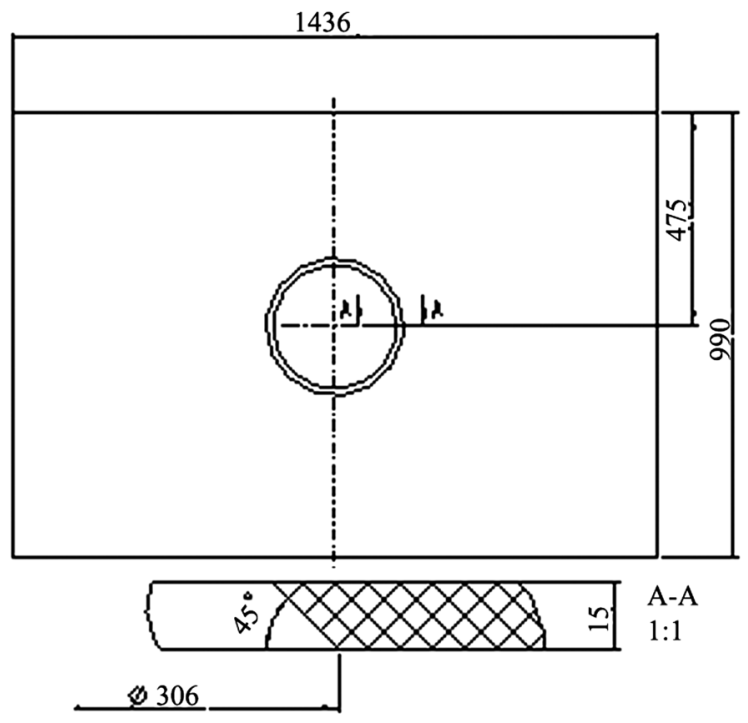

FIG. 10. Drawing of the acrylic insulator, installed between stages. and beam energy. Good agreement was observed between current $I 0$, measured in the beginning of a line, and the diode current $I 1$, which proves that there is no leakage in the vacuum coaxial at the electric field at the cathode up to

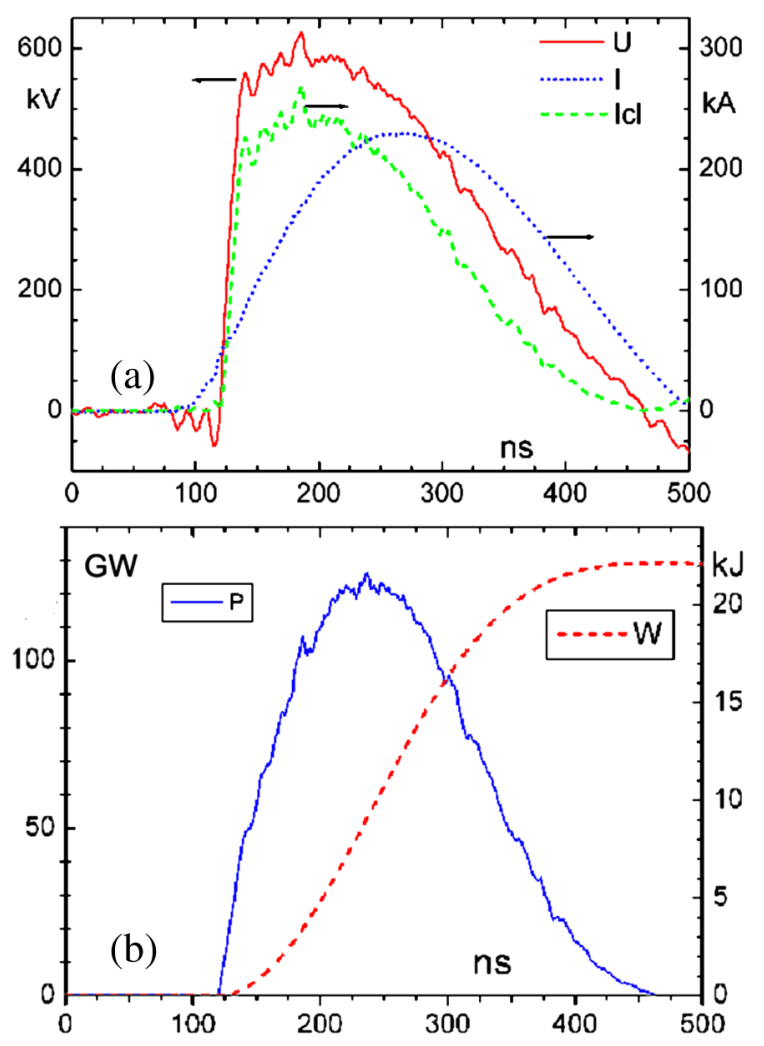

FIG. 11. (a) Waveforms of the diode voltage $U$, diode current $I$, and one-dimensional space charge limited current $I c l$ at $95 \mathrm{kV}$ charging voltage. (b) Calculated traces of the diode power $P$ and energy $W$. 
$234 \mathrm{kV} / \mathrm{cm}$. Synchronization and triggering systems provide synchronous firing of all 24 LTD stages of the accelerator.

The next parameters of the accelerator have been obtained: diode voltage- $550-600 \mathrm{kV}$; diode current276-230 kA; current rise time-160 ns; maximum power of the electron beam-130 GW; pulse width on half maximum-160 ns; electron-beam energy at power level not less than half of maximum value $-20 \mathrm{~kJ}$. The total energy of electrons, which pass through a $40 \mu \mathrm{m}$ Ti foil into the Xe cell, is $8-9 \mathrm{~kJ}$ in the $150-160 \mathrm{~ns}$ pulse (full width at half maximum), mean specific power of energy input into gas cavity is $\sim 330 \mathrm{~kW} / \mathrm{cm}^{3}$. Typical waveforms for $95 \mathrm{kV}$ charging voltage are given in Fig. 11. About 1000 shots have been done in tests of this accelerator. From the middle of 2011 it operates on a daily basis in laser experiments with shot rate required for those experiments, and the total amount of shots exceeds 3000 up to now. The only failure during tests was breakdown in one of charging cables.

\section{CONCLUSION}

A new approach in LTD technology was introduced and proved experimentally. Developed stages and installations on their base have shown good performance, reliability, and robustness. Only dry air at atmospheric pressure is required as a spare supply for the new LTD stage. Design concepts, developed for these stages and installations, can be easily replicated in the design of multi-MV and multiMA generators. Further experimentation is planned with the lifetime tests and capacitor block modification.

[1] A. N. Bastrikov, V. A. Vizir, S. N. Volkov, V. G. Durakov, A. M. Efremov, V. B. Zorin, A. A. Kim, B. M. Kovalchuk, E. V. Kumpyak, S. V. Loginov, V. A. Sinebryukhov, N. V. Tsou, V. V. Chervyakov, V.P. Yakovlev, and G. A. Mesyats, Laser Part. Beams 21, 295 (2003).

[2] W. A. Stygar, M. E. Cuneo, D. I. Headley, H. C. Ives, R. J. Leeper, M. G. Mazarakis, C. L. Olson, J. L. Porter, T. C.
Wagoner, and J.R. Woodworth, Phys. Rev. ST Accel. Beams 10, 030401 (2007).

[3] T. Ao, J. R. Asay, S. Chantrenne, M. R. Baer, and C. A. Hall, Rev. Sci. Instrum. 79, 013903 (2008).

[4] A. V. Kharlov, B. M. Kovalchuk, and V. B. Zorin, Rev. Sci. Instrum. 77, 123501 (2006).

[5] I. D. Smith, Phys. Rev. ST Accel. Beams 7, 064801 (2004).

[6] A. A. Kim, M. G. Mazarakis, V.A. Sinebryukhov, B. M. Kovalchuk, V.A. Visir, S. N. Volkov, F. Bayol, A. N. Bastrikov, V. G. Durakov, S. V. Frolov, V. M. Alexeenko, D. H. McDaniel, W.E. Fowler, K. LeChien, C. Olson, W. A. Stygar, K. W. Struve, J. Porter, and R.M. Gilgenbach, Phys. Rev. ST Accel. Beams 12, 050402 (2009).

[7] M. G. Mazarakis et al., IEEE Trans. Plasma Sci. 38, 704 (2010).

[8] B. M. Kovalchuk, A. V. Kharlov, A. A. Zherlitsyn, E. V. Kumpiak, N. V. Tsoy, V.A. Vizir, and G. V. Smorudov, Laser Part. Beams 27, 371 (2009).

[9] A. A. Zherlitsyn, B. M. Kovalchuk, G. V. Smorudov, N. V. Tsoy, V. A. Visir, and V. B. Zorin, in Proceedings of the 15th International Symposium on High Current Electronics, edited by B. Kovalchuk and G. Remnev (Institute of High Current Electronics SB RAS, Tomsk, Russia, 2008), p. 296 [http://www.hcei.tsc.ru/conf/2010/ cat/proc_2008/shce/296-298.pdf].

[10] B. M. Kovalchuk, A. V. Kharlov, V. B. Zorin, and A. A. Zherlitsyn, Rev. Sci. Instrum. 80, 083504 (2009).

[11] A. A. Zherlitsyn, B. M. Kovalchuk, and G. V. Smorudov, Instrum. Exp. Tech. 52, 802 (2009).

[12] S. I. Braginskii, Sov. Phys. JETP 34, 1068 (1958).

[13] S. I. Andreev and B. I. Orlov, Sov. Phys. Tech. Phys. 30, 1097 (1966).

[14] A. V. Kharlov, B. M. Kovalchuk, E. V. Kumpyak, A. A. Zherlitsyn, V. B. Zorin, G. V. Smorudov, F. Bayol, and F. Lassalle, in Proceedings of the 16th International Symposium on High Current Electronics, edited by B. Kovalchuk and G. Remnev (Institute of High Current Electronics SB RAS, Tomsk, Russia, 2010), p. 261 [http:// www.hcei.tsc.ru/conf/2010/cat/proc_2010/shce/261-264.pdf].

[15] B. M. Kovalchuk, A. V. Kharlov, S. N. Volkov, A. A. Zherlitsyn, V.B. Zorin, G. V. Smorudov, and V. N. Kiselev, Laser Part. Beams 30, 23 (2012). 